\title{
Creation and Deployment of a Virtual, Inquiry-Guided Biomedical Engineering Laboratory Course
}

\author{
Casey J. Ankeny (1) and Matthew C. Tresch (i) \\ Biomedical Engineering, Northwestern University, Evanston, IL, USA \\ (Received 24 June 2020; accepted 5 August 2020; published online 18 August 2020)
}

\section{CHALLENGE STATEMENT AND MOTIVATION TO INNOVATE IN A REMOTE SETTING}

The rapid switch to online instruction in Spring 2020 due to the COVID-19 pandemic presented a unique challenge for laboratory courses and jeopardized the mastery of many learning objectives. In particular, our sophomore-level experimental design and measurement laboratory course needed to be effectively adapted prior to the start of the Spring quarter to provide biomedically-relevant, hands-on experience with measurement, hypothesis generation, experimental design, and team collaboration in a remote environment. Additionally, new challenges arose as we moved to an online platform including maintaining engagement, enabling interactions between students, and peer teaching.

\section{NOVEL INITIATIVE}

Overview of Innovation

To address these challenges, we created a twomodule, team-based course where students participated in scaffolded- and inquiry-guided experiences ${ }^{2}$ about sensor characterization and experimental design in a biomedically-relevant context. Moreover, this 0.5unit, quarter-long course provided opportunities for community development in an online platform by requiring students to work within and across teams. This course design leveraged several evidenced-based practices including the flipped classroom ${ }^{9,13}$ featuring mini-lectures and pre-lab activities, a pre-/post-quiz to

Address correspondence to Casey J. Ankeny, Biomedical Engineering, Northwestern University, Evanston, IL, USA. Electronic mail: casey.ankeny@northwestern.edu assess students' knowledge and tailor the instruction, ${ }^{12}$ standards-based grading based on a problem-solving skillset, ${ }^{4}$ and team-building exercises including ice breakers and team charters. ${ }^{11}$

\section{General Course Structure featuring Synchronous and Asynchronous Components}

We first describe the overall structure of the course and how synchronous and asynchronous components were organized over the term. To encourage sustained engagement, students completed pre-lab work consisting of a few short online lectures and an activity to apply the lectures' concepts. Additionally, students submitted unclear points (Muddiest Points) ${ }^{3}$ anonymously through the course management software (Canvas) one day prior to the synchronous lab period. The instructional team then reviewed the activity responses as well as the Muddiest Points submissions. The instructional team posted the Muddiest Point responses on the course's discussion board the evening before the lab session.

The synchronous lab session began with a brief review of the Muddiest Points as well as a description of the session's goals via Zoom. Students then worked in their own team's Zoom spaces. The module instructor and the two teaching assistants each had a virtual office (i.e., their own Zoom room) throughout the synchronous session. Student teams would drop in to the virtual offices as needed. Moreover, each team had two scheduled meetings - one with a teaching assistant and one with the module instructor-during each lab period. The class reconvened at the end of the session via Zoom to discuss important points that arose during the period and to give an overview for the next week. 


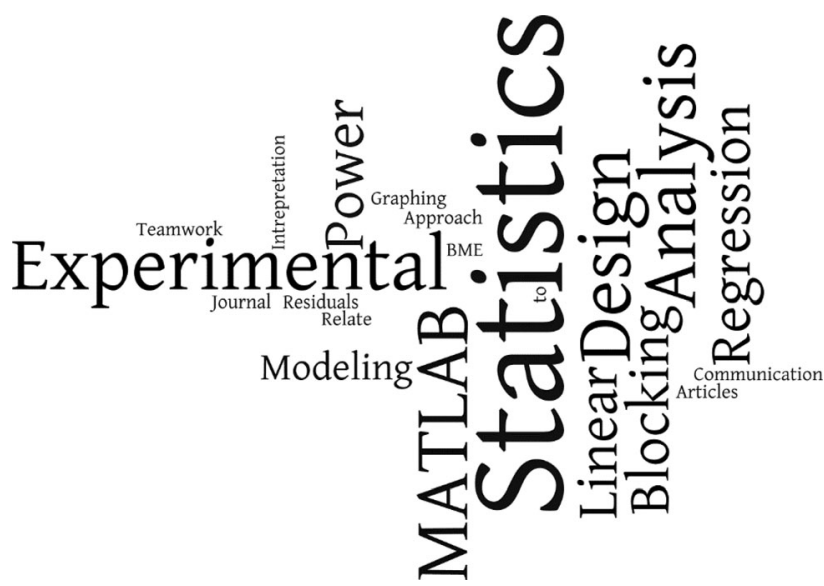

FIGURE 1. Open-ended student responses about acquired skills $(n=13)$.

\section{Virtual Team Building}

The instructors formed groups by time zones to facilitate meeting outside of the synchronous lab session. Groups were also balanced with respect to sex. To provide opportunities for the development of community and team-building online, we included an ice breaker on the first day of the course. Teams completed team charters at the beginning of each module ${ }^{11}$ that included questions about how conflicts would be handled as well as special considerations for sickness and the remote learning environment. Student teams listed challenges that they anticipated with virtual lab instruction that were then discussed with the entire class and instructional team and incorporated into course design. Both the ice breaker and team charter may be found in the supplemental materials.

As mentioned above, students worked in their own Zoom spaces and had regular meetings at the classand group-levels to facilitate interactions as well as peer teaching. Outside of the synchronous lab session, teams met primarily through GroupMe, Facebook, and Zoom. Additionally, teams were regularly assessed using peer and team evaluation as described in the supplemental materials. Not only did students have the opportunity to work in teams but they also worked across teams in Module 2. Student teams remained constant throughout the term and all students voluntarily served as participants in other teams' experiments.

\section{Course Learning Objectives and Standards-Based Grading}

The course design was guided by the established learning objectives pertaining to sensor characterization (Module 1) and experimental design (Module 2). The instructors assessed students in the development of problem solving skills in both modules. ${ }^{6,1}$ More specifically, lab drafts and final reports were graded with a mastery-based grading rubric in the following focus areas: problem identification, knowledge processing, experiment design/approach, analysis, interpretation, teamwork, and communication. The grading rubric and templates for the drafts and reports may be found in the supplemental materials.

In the first module, students focused on understanding sensor properties, calibration, and propagation of uncertainty. They created sensors to measure personal activity levels and characterized them in terms of accuracy, precision, discrimination, and sensitivity. In the second module, students devised experiments to investigate differences in heart rate among experimental groups. They incorporated elements of a welldesigned experiment such as controls, blocking for confounding factors, conducting power analyses using $\mathrm{G}^{*}$ Power, ${ }^{7}$ and randomization. Moreover, they used the literature to generate hypotheses with biomedical significance and trained other teams to collect data for their experiment. Students then analyzed the data using models that accounted for confounding factors by blocking as fixed or random effects. The supplemental materials include the course syllabus, learning objectives, and weekly class layouts for both modules.

\section{Collection and Interpretation of Biomedically-Relevant Data Using Apps and Cell Phones}

To address the needs for hands-on experience with taking measurements in a team-based setting, teams collected their own data remotely using readily available apps that work well with a variety of cell phones. In Module 1, students conducted two activities. In the first activity, teams created and characterized a sensor to measure distance from a light source using the Physics Toolbox ${ }^{10}$ and Galactica Lux Meter. ${ }^{8}$ In the second activity, students used the MATLAB mobile app to create a sensor that measured activity levels (both steps counted and calories burned). To increase engagement, teams competed against the instructor by comparing whose algorithm was best at determining step number. Additionally, this activity was motivated by a guest lecturer, Dr. Arun Jayaraman, who spoke about validating activity monitors for rehabilitation.

In Module 2, students gained the desired experience with hypothesis creation and experimental design. Specifically, they used the readily available heart rate monitoring app, Cardiograph ${ }^{5}$ to collect data at home. This technology used the phone's light source, photodetector, and photoplethysmography to measure volumetric variations of blood circulation to determine heart rate. Each team determined their own hypothesis using peer-reviewed literature, crafted an experimental 
design with help from the instructional team, created a video to describe how other teams should collect data, collected and analyzed these data, and wrote a laboratory report explaining their findings. Handouts for both modules may be found in the supplemental materials.

\section{REFLECTION}

\section{Assessment of Skill Development in a Remote Setting}

To gauge mastery of the course objectives in this remote setting, students were graded with a standardsbased grading rubric focusing on problem solving. ${ }^{6,1}$ All teams mastered the course standards as demonstrated by lab draft and report submissions. In addition, students completed a course survey $(n=13$ out of 15) outlined in the supplemental materials. When prompted about the skills learned in the course, students cited many desired outcome topics as seen in Fig. 1 where the size of the word indicates frequency. Popular responses included statistics, experimental design, and MATLAB.

\section{Qualitative Student Feedback About the Students' Experience}

Open-ended course survey responses $(n=13)$ about their experience in this remote offering provided organic student feedback about how well the course implemented aspects to address the aforementioned challenges of a remote laboratory course. In particular, we learned about the students' attitude with respect to the class structure; team aspects such as ice breakers, charters, peer and team evaluation as well as modes of communication; enthusiasm for biomedical engineering; and preparation for research.

The students identified strengths and weaknesses of the course structure. Five of the eight students who mentioned time allocation felt that certain lectures and experimental periods were too long. Eight of the 13 students thought that the workload matched the credit amount. All students that mentioned the activities found them favorable $(n=3)$. Overall students reacted positively to the course structure as highlighted by two representative quotes:

- "I think everything was balanced well. The pre class lectures were good for gaining more in depth knowledge on what we would be doing in class and learning the MATLAB tools."

- "The activities were very balanced, and it was nice to learn some concepts outside of class so we had time to prepare for in class work. Meeting at the beginning of each class was good to recap important concepts and get a more in-depth overview of the assignment."

The analysis of the team and peer evaluations after each module as well as the course survey uncovered functional teams in this virtual setting. Students appreciated the initial ice breaker activity. One student stated that the "[f]irst meeting was very conducive to this atmosphere so thank you to the professors for using the first week to build healthy team dynamics before jumping into lecturing straight away." Moreover, all peer evaluations were positive with the exception of one student being quiet but a hard worker. When asked to evaluate team's communication, all four teams in both modules stated that their team communicated effectively. One student stated that "[w]e are all comfortable voicing our concerns and ideas. A concrete example is when we settled a 'coding dispute' by presenting the argument for each side civilly and voting at the end to decide which route should be ultimately taken to achieve an algorithm that everyone's happy with." The authors attributed this successful conflict resolution in part to the team charter that outlined steps for this situation. One student highlighted that refinement of virtual communication is important not only during the COVID-19 pandemic but beyond: "The virtual team working skillset is also pretty valuable as the world shifts more to the digital space in the future."

Overall, students felt that the course increased their enthusiasm for biomedical engineering (12 of 13 responses). One student stated that the course "connect[ed] the specific labs [in the course] and their influence in the world of BME/health" and that "helped...understand more of the influence BME has in industry." Students also liked the guest mini-lecture from Dr. Arun Jayaraman. One student called for additional lectures of this nature: "The interview with the researcher was interesting and I'd like to see more tangents like that. I think that by drawing more of these connections, the course would feel more like BME and less like stats."

Students also thought that the course prepared them for research. Four of the five students who were already engaged in research said that the skills they learned in this virtual lab course would help in their future research. Seven of the eight students that were not currently engaged in research stated that this course made them feel more confident to perform research. The authors were encouraged by this result because a major course objective is to prepare student for research earlier in their undergraduate career. 


\section{DISCUSSION AND FUTURE WORK}

Future iterations of this remote laboratory course would benefit from contingency plans for students unable to attend synchronous lab sessions or living in different time zones from their team members. For example, synchronous sessions could be recorded and posted to the course management site. Further, teams could adapt meeting times amongst themselves and with the instructional team to better suit availability and technological constraints. Data collection can continue outside of class sessions asynchronously.

In the event of scaling this course to a larger cohort, special consideration must be made to ensure there is ample instructional support as many aspects of the course benefited from the 5:1 ratio of students to instructor/teaching assistants. In the event ratios of students to instructional team members are higher, the instructors may consider reducing the instructional meetings per lab session from two to one as well as offering additional office hours outside of class time. Instructors also may consider meeting with multiple groups at the same time and encouraging more intergroup collaboration and community. For example, instructors may consider replacing one meeting with a group-to-group meeting, rather than a meeting with the instructor or TA, to allow for peer mentoring. In larger class sizes with multiple graders, grader calibration will be important.

Although we did not encounter students without a smartphone, we must consider how students may engage in these modules without access to a smartphone or reliable internet connections. Considering technology constraints would be wise during team formation to ensure each team has access to a smartphone as well as during team charter discussions to make sure each member has the opportunity to participate, even if they have technological challenges.

Also, in future course iterations the authors will better guide the reflection activities to allow for deeper understanding of individual progress towards developing the problem solving standards. Moreover, the authors will recreate the course videos such that every video has the instructor's video stream as well as embedded pop-up questions to better engage the viewer. A more quantitative analysis will be conducted to determine which aspects worked well and those that needed improvement from the student perspective.

\section{CONCLUSION}

Overall, this course allowed students to participate in team-based laboratory modules focusing on sensor characterization and experimental design. The course design included many evidence-based practices such as the flipped classroom where short online lectures were coupled with pre-lab activities, standards-based grading centered on problem solving, formative feedback informed by the Muddiest Point exercise, and a guided-inquiry laboratory module to address the challenges apparent in an online laboratory course. This course allowed students to collect biomedically-relevant data at home in a setting that fostered a sense of community. Overall students viewed the remote adaption of this experiment design and analysis laboratory course favorably. Students were "happy with the range that the course covered". Further, some students would like to see these modules used even when we return to campus as explained in the following quote: "I think given the circumstances of remote learning, the experiments we did were very applicable in our own homes and most importantly, doable. Going forward I think they could be intro experiments for students on campus to do before they begin more advanced experiments with the complicated devices."

\section{ELECTRONIC SUPPLEMENTARY MATERIAL}

The online version of this article (https://doi.org/10. 1007/s43683-020-00017-w) contains supplementary material, which is available to authorized users.

\section{ACKNOWLEDGMENTS}

The authors would like to acknowledge David O'Neill and Eric Perreault for the development of the course survey. We would also like to acknowledge the course's teaching assistants, Maggie Swerdloff and Ege Altan.

\section{AUTHOR CONTRIBUTIONS}

Both CA and MT developed the course. CA drafted the manuscript. MT revised the manuscript evaluating it critically for intellectual content.

\section{FUNDING}

Not applicable.

\section{CONFLICT OF INTEREST}

Not applicable. 


\section{REFERENCES}

${ }^{1}$ Ankeny C, O'Neill D. Work in progress: aligning and assessing learning objectives for a biomedical engineering course sequence using standards-based grading within a learning management system. American Society of Engineering Education, 2019.

${ }^{2}$ Beck C, Lawrence B. Inquiry-based ecology laboratory courses improve student confidence and scientific reasoning skills. Ecosphere. 2012;3(12):1-11.

${ }^{3}$ Carberry A, Krause S, Ankeny C, Waters C. 'Unmuddying' course content using muddiest point reflections. IEEE Front. Eng. Educ. 2013.

${ }^{4}$ Carberry A, Siniawski M, Atwood S, Diefes-Dux H. Best practices for using standards-based grading in engineering courses best practices for using standards-based grading in engineering. ASEE Conf. Proc. 2016.

5"Cardiograph - Heart Rate Meter." [Online]. https://play.g oogle.com/store/apps/details?id = com.macropinch.hydra.a ndroid\&hl $=$ en_US.

${ }^{6}$ Edwards-Schachter M, SanchezRuiz LM, Sanchez-Diaz LM. Achieving competence-based curriculum in engineering education in Spain. Proc IEEE. 2009;97(10):1727-36. h ttps://doi.org/10.1109/jproc.2009.2026064.
${ }^{7}$ Faul AG, Erdfelder F, Buchner E, Lang A. Statistical power analyses using $G^{*}$ Power 3.1: tests for correlation and regression analyses. Behav Res Methods. 2009;41:1149-60.

"'Galactica Luxmeter." [Online]. https://apps.apple.com/u s/app/galactica-luxmeter/id666846635.

${ }^{9}$ Mason G, RutarShuman T, Cook KE. Comparing the effectiveness of an inverted classroom to a traditional classroom in an upper-division engineering course. Educ IEEE Trans. 2013;56:430-5.

10“"Physics Toolbox Sensor Suite." [Online]. https://play.go ogle.com/store/apps/details?id = com.chrystianvieyra.physi cstoolboxsuite\&hl = en US.

${ }^{11}$ Rice E, Smedick W. Tools for creating and managing student teams. Washington: American Society of Engineering Education; 2018.

${ }^{12}$ Rolfe I, McPherson J. Formative assessment: how am I doing? Lancet. 1995;345(8953):837-9.

${ }^{13}$ Wilson SG. The flipped class: A method to address the challenges of an undergraduate statistics course. Teach Psychol. 2013;40(3):193-9.

Publisher's Note Springer Nature remains neutral with regard to jurisdictional claims in published maps and institutional affiliations. 